\title{
Internet interventions for improving psychological well-being in psycho-oncology: review and recommendations
}

\author{
Yan Leykin', Seema M. Thekdi², Dianne M. Shumay ${ }^{1,3}$, Ricardo F. Muñoz ${ }^{1,4}$, Michelle Riba ${ }^{5}$ and Laura B. Dunn 1,3* \\ 'Department of Psychiatry, University of California, San Francisco, CA, USA \\ ${ }^{2}$ Department of Psychiatry, M.D. Anderson Cancer Center, Houston, TX, USA \\ ${ }^{3}$ UCSF Helen Diller Family Comprehensive Cancer Center, University of California, San Francisco, CA, USA \\ ${ }^{4}$ San Francisco General Hospital, San Francisco, CA, USA \\ ${ }^{5}$ Department of Psychiatry, University of Michigan, Ann Arbor, MI, USA
}

* Correspondence to: Department of Psychiatry, University of California, San Francisco, 401 Parnassus Avenue, Box 0984-F, San Francisco, CA 94 143-0984, USA E-mail: Laura.Dunn@ucsf.edu

Received: 12 February 2011 Accepted: I| April 20I I

\begin{abstract}
Objective: Too few cancer patients and survivors receive evidence-based interventions for mental health symptoms. This review examines the potential for Internet interventions to help fill treatment gaps in psychosocial oncology and presents evidence regarding the likely utility of Internet interventions for cancer patients.

Methods: The authors examined available literature regarding Internet interventions tailored to cancer patients' mental health needs and reviewed elements of Internet interventions for mental health relevant to advancing psycho-oncology Internet intervention research.

Results: Few rigorous studies focusing on mental health of cancer patients have been conducted online. A growing body of evidence supports the efficacy, accessibility, and acceptability of mental health Internet interventions for a variety of general and medical patient populations. The authors present recommendations and guidelines to assist researchers in developing, testing, and disseminating Internet interventions for cancer patients and survivors, to manage and improve their mental health. Issues unique to Internet interventions-including intervention structure, customization, provider interaction, and privacy and confidentiality issues-are discussed. These guidelines are offered as a step toward establishing a set of "best practices" for Internet interventions in psycho-oncology and to generate further discussion regarding the goals of such interventions and their place in cancer care.

Conclusions: Internet interventions have the potential to fill an important gap in quality cancer care by augmenting limited available mental health services. These interventions should be developed in a manner consistent with best practices and must be empirically tested and validated.
\end{abstract}

Copyright (C) 2011 John Wiley \& Sons, Ltd.

Keywords: cancer; oncology; intervention studies; Internet; depression; anxiety; quality of life; research methods; Internet intervention

\section{Introduction}

Patients diagnosed with cancer universally experience at least some level of distress. Feelings of fear, grief, and loss can occur at different phases of illness, including diagnosis, active treatment, recurrence, and transition to survivorship or palliative treatment. Approximately $20 \%$ to $40 \%$ of oncology patients develop depression [1-3]. Similar prevalence rates are reported for anxiety symptoms, including overall health concerns, monitoring-related anxiety, fears about recurrence/progression, post-traumatic stress symptoms, and worries about pain and death [4-8]. Depression and anxiety negatively affect quality of life, adherence, costs, health behaviors, and decision making $[1,9,10]$. Several randomized controlled trials have shown that depression can be treated effectively in cancer patients [11-15]. Although fewer interventions addressing anxiety in cancer patients have been examined in randomized controlled trials, these also show promise [16-18].

Inadequate screening for psychological distress, and, specifically, for depression and anxiety, remains an area of concern in cancer care $[1,19,20]$. Even when undertaken, screening alone has not proven effective in leading to increased mental health referrals or treatment utilization $[19,21,22]$. Reasons for this include lack of trained mental health professionals, insurance coverage and cost issues, stigma and privacy concerns associated with receipt of mental health services, and geographical distance from providers [23]. Consequently, far too few patients receive evidence-based interventions for mental health symptoms [1], and many patients report that their emotional needs go unmet [19].

Inadequate screening and referral, and barriers to psychosocial care for cancer patients highlight the need to develop and test new treatment models 
that can help fill these service gaps. Internet mental health interventions have the potential to help meet the needs described above and help reduce existing mental health disparities [24]. Indeed, when faced with the emotional stress, difficult decisions, and physical challenges that accompany their cancer experience, many patients search for support online [25].

Internet interventions-evidence-based, empirically tested psychological interventions-may therefore have broad reach and acceptability to patients and families. This review examines the potential for Internet interventions to reduce treatment gaps; describes important issues in the development, empirical testing, and dissemination of such interventions; and outlines a set of recommendations and guidelines for Internet mental health intervention research tailored to cancer patients.

\section{Internet interventions for mental health}

\section{Available Internet resources for cancer patients}

There are at least three types of Internet health resources.

(i) Information sites

(ii) Mutual support sites

(iii) Evidence-based Internet interventions

Currently, the vast majority of available Internet resources for cancer patients consist of informational websites and online mutual support groups. Though these resources can offer support and validation, they are not designed to actually treat and ameliorate the symptoms of psychiatric distress, such as depression and anxiety. On informational websites, patients can find basic information about symptoms, followed by a list of general suggestions, or advice to "talk to your doctor about [specific symptom]." Some suggestions may have the unintended effect of making some patients feel worse, such as when a recommended strategy is ineffective or harmful, or too difficult or expensive, or when ineffective coping styles are inadvertently reinforced through reassurance [26].

Online support groups may help individuals feel validated, and personal narratives on social media allow for sharing of experiences among cancer patients [27]. However, these modalities do not provide evidence-based therapies [28], and research on the effects of online support groups suffers from methodological limitations [29,30]. Patients with significant depression or anxiety need more than information and support; they need to learn and practice empirically supported methods of behavior change. Such skills can be taught online, as demonstrated by a growing body of research on Internet interventions [31].

\section{Potential benefits of Internet mental health interventions in cancer patients}

Internet interventions have the potential to fill an important gap in quality cancer care by adding to limited available mental health services [32-34] and offering valuable tools to help people better manage the emotional side of cancer treatment.

\section{Acceptability}

Evidence-based Internet interventions can offer customizable, engaging access to mood and anxiety management tools [35]. Online learning and interactive communication tools are well received by patients, including cancer patients, many of whom already seek information and support online [19]. Patients who are actively engaged in their care also may be more likely to learn, retain, and use the new tools-effects possibly mediated through greater self-efficacy [36]. Because the receipt of psychological therapies continues to be highly stigmatized in many subcultures, an additional benefit of Internet interventions is greater privacy and confidentiality. Individuals can seek treatment at home at their convenience, an important aspect for patients overwhelmed by frequent medical appointments.

\section{Accessibility}

Internet interventions are particularly well suited to reducing health disparities. They are scalable-that is, accessible by many people, simultaneously and repeatedly [37]. Face-to-face clinician encounters, support groups, medications, and virtually all other methods are consumable - only one person (or one group) benefits at one time [24]. A visit to an Internet intervention website does not use up the resource nor does it prevent another patient or another thousand patients from using it at the same time. In addition, inperson interventions, once disseminated, may lose fidelity because of differences in training, time pressures, or even personal philosophy of providers. In contrast, Internet interventions remain perfectly consistent. This consistency does not mean stagnation, however, as Internet interventions can be rapidly updated with new information and tools, as well as expanded into other languages.

\section{Efficiency}

The Internet is arguably the most time-effective and cost-effective method of intervention delivery. In the USA, psychological services-particularly those delivered by experienced psycho-oncology providers-are relatively scarce outside urban areas and may be prohibitively expensive. For cancer patients, many of whom already struggle with expenses associated with treatment and disability, psychological care can easily fall by the wayside. Internet interventions could provide access to inexpensive or free treatment options. 


\section{Enhanced quality of care}

Internet interventions can offer useful tools for oncology providers, given limitations on their time as well as limited availability of mental health providers. The ability to refer patients to trusted, evidence-based intervention websites for help with psychological aspects of cancer would be a valuable adjunct to clinical care. Many Internet interventions contain interactive progress trackers, which could enable clinicians to monitor their patients' symptom status.

\section{Public health impact}

Given the dearth of mental health services available to the majority of cancer patients, Internet interventions are unparalleled in their potential impact from both a clinical oncology and a public health perspective. Even if the effect on each individual person is smaller than for therapy conducted by a live clinician, small improvements in a larger group of patients may contribute a great deal to public health and may be an excellent use of resources-for example, as in RE-AIM [38]. Importantly, Internet interventions can offer the same care to anyone, including populations that are typically underserved [39], without necessitating changes in local health care systems or financing. As Internet interventions evolve, they can be translated into languages other than English [40], further increasing access.

\section{Limitations of Internet mental health interventions in cancer patients}

Internet interventions also carry limitations that must be acknowledged.

\section{Internet access constraints}

Utilizing an Internet intervention requires Internet access and basic computer literacy. Thus, Internet interventions may systematically exclude those from lower socioeconomic strata. However, Internet penetration is already at over $77.3 \%$ in the USA and is rapidly increasing in the rest of the world [41].

\section{Limited personal interaction}

Internet interventions, regardless of their complexity, cannot replace the personal touch, adaptability, and flexible treatment of a live clinician. Internet interventions cannot offer empathy to the patient, react to patients' non-verbal cues, confusion, or treatment-compromising behaviors and attitudes, or offer additional support if treatment is not going well. Most (but not all) Internet interventions offer only pre-programmed material and cannot ask follow-up questions about the patient's unique circumstances or address unexpected comorbidities. With Internet mental health interventions, there is limited ability to respond in case of a crisis (although hotlines and other crisis intervention resources can and should be provided). Thus, regardless of how sophisticated an Internet intervention might be, it cannot fully replace a live clinician and should not be considered as a replacement for inperson psychological care, where available and accessible.

\section{No therapeutic contract}

Internet interventions for mental health have also raised ethical concerns regarding therapeutic contracts and ultimate responsibility for patients' welfare. Internet interventions are best viewed as self-help resources, akin to bibliotherapy. The reader of a self-help book, while hoping that the book will be beneficial, understands that there is no therapeutic contract between himself or herself, the author, or the publisher. Similar understanding must be in place for any ethically sound Internet intervention. Specifically, those who design, test, and disseminate such interventions need to make explicit (and, as underscored in the Research section, test for participants' understanding) that the intervention is self-help, that benefits for any given individual are not guaranteed, that users' responses are not reviewed in real time, and that for any emergencies, a qualified professional must be sought.

\section{Evidence of effectiveness of Internet mental health interventions for cancer patients}

A limited database is available on Internet interventions specific to cancer patients. In contrast, a number of such interventions have been developed targeting depression and anxiety in general and other medical populations [42-45]. With few exceptions, most do not provide interactive tools to link patients and providers [46,47]. One self-guided, online cognitive behavioral therapy (CBT) coping-skills group intervention for early stage breast cancer focused on symptoms, with health-related quality of life as the outcome [47]. This small study $(n=62)$ found no main treatment effects after 12 weeks, although those participants in the treatment condition who had lower self-rated health status at baseline reported greater improvement. Several Internet symptom management interventions are being studied to help patients with specific types of cancer, such as the WRITE Symptoms intervention for ovarian cancer, delivered by nurses via Internet message boards [48]. A randomized trial $(n=405)$ of a telecare symptom management program for cancer patients with depression and/or pain, which utilized an Internet-based symptom monitoring system as a component of the intervention [49], reported improvements in both pain and depression. Another recently completed pilot study used an Internet intervention (SHUTi-C) to address insomnia in cancer patients [50].

Online support groups, while popular, are not supported by empirical evidence regarding their 
efficacy [51]. A recent report of a randomized controlled trial of a newly diagnosed breast cancer Internet peer support group found that the peer support condition resulted in worse outcomes in terms of distress and quality of life [52]. A subsequent study involving cancer survivors found a transient difference in mood and adjustment scores, but sustained benefit was not confirmed [29]. However, a study of another, moderated online support group for breast cancer patients $(n=72)$ reported decreases in depression, perceived stress, and cancer-related trauma, with moderate effect sizes [46].

Taken together, these studies, although limited in number and heterogeneous in methods and measures, suggest the feasibility and acceptability of Internet interventions for both psychological and physical symptoms in patients with cancer, while providing mixed evidence for effectiveness. Moreover, the studies underscore the need for methodologically rigorous studies both to establish the efficacy and to identify which interventions are best suited for which patients.

\section{Recommendations for the development of psycho-oncology Internet interventions}

\section{Developing empirically validated Internet interventions}

Preventive and treatment interventions should be supported by empirical research. Most of the traditional face-to-face mental health interventions offered today have strong empirical support [15,53-56]. If Internet interventions are to join the ranks of bona fide preventive and treatment methods for emotional distress for cancer patients and survivors, they must undergo similarly rigorous testing to achieve the status of empirically supported Internet interventions. The need to bring scientific rigor to online behavioral interventions was outlined in a statement from the International Society for Research on Internet Interventions [57], as well as by other researchers [58,59].

Some of the commonly applied criteria for establishing empirical support may not apply to interventions delivered online. For instance, to determine the efficacy of a treatment, a common criterion is superiority or at least equivalence to an established efficacious treatment, as demonstrated by two or more trials, conducted by two or more separate research teams. It is unlikely that two independent research teams will develop and test exactly the same Web program. But two teams could test the same site (or compare sites developed by each team) on their own patient populations. Other criteria must continue to be held to the highest standards. These include a clear theoretical rationale for treatment and methodological rigor in establishing efficacy. "Active ingredients" of specific interventions can be examined through dismantling studies.
Methodological rigor will also include, among other considerations, careful pre-screening (although not necessarily exclusion, to preserve ecological validity) of participants for pre-morbid (i.e., pre-cancer) psychiatric and medical disorders and pre-morbid psychological coping strategies (to establish new learning), as well as pre-screening for other potentially important factors, such as personality, health-related beliefs, or self-efficacy.

\section{Developing guidelines and best practices for Internet} interventions in psycho-oncology

Designing Internet interventions to benefit oncology patients necessitates familiarity with common intervention components, as well as appreciation of issues specific to the development of Internet interventions. Below, we define and discuss key elements of such interventions, with the goal of beginning to establish best practices for Internet interventions for psycho-oncology.

\section{Elements particular to Internet interventions}

Accurately defining the components and the process of Internet intervention is necessary for the development of a research agenda for such mental health interventions tailored to psycho-oncology needs.

\section{Evidence-based Internet interventions}

As recently operationalized by Barak et al. [60], an Internet intervention is

a primarily self-guided intervention program that is executed by means of a prescriptive online program operated through a website and used by consumers seeking health- and mental-health related assistance. The intervention program itself attempts to create positive change and or improve/enhance knowledge, awareness, and understanding via the provision of sound health-related material and use of interactive web-based components.

Equally important is clarifying what Internet interventions are not. Specifically, "static" information-only websites, or sites offering medical advice inconsistent with currently established and empirically based best practices, are not considered evidence-based Internet interventions by the proposed definition.

\section{Skill building}

Skills training, a key component of Internet interventions, involves building resources to cope with the numerous psychological and physical aspects of cancer diagnosis, treatment, and survivorship. These could include tools to help patients manage anxiety and depressive symptoms and other emotional and physical symptoms prevalent in oncology populations, such as fatigue, sleep disturbance, pain, and 
body image issues, among others. A crucial component of skill building is homework, or practice, which is known to improve outcomes [61,62]. A quality skill-building component would therefore include an opportunity to practice new skills, for instance, via an interactive worksheet or tool or an explicit homework task or assignment. Such skills could include mood monitoring, pain management, tracking activity levels, behavioral activation, tracking and working with mood-related thoughts, among others.

\section{Personalization/tailoring}

"Tailoring" implies that the information and feedback presented is specifically relevant to the individual patient, and all other information the website is capable of providing is suppressed. In practice, tailoring may range from providing customized feedback based on the individuals' calculated score on completed measures (e.g. "Your level of pain/discomfort is $[\mathrm{X}]$, which is lower than the last time you were here-that's good news!"), to comprehensive procedures that might involve generating an "avatar" personality that looks like a participant and provides immediate feedback based on past and present site activities. Some level of customization may be beneficial for two reasons: it may help the patient feel more understood and perceive a greater relevance of the program [63], and it reduces the burden of information that is irrelevant to the specific diagnosis or stage of cancer (e.g., those with prostate cancer, but not with breast cancer, would be provided with information on continence).

\section{Tracking}

A system for participants to track their symptoms and tool use is important. For patients, symptom and mood monitoring may be associated with greater treatment involvement [64]. An additional benefit of symptom and mood monitoring is the ability of the patient to be educated regarding the relationship of their actions (e.g., doing pleasant activities, responding to automatic thoughts, etc.) to psychological well-being, as is commonly practiced in live therapy. With some websites, tracking may also enable a medical provider to be aware of the patient's progress and current status. Such tracking may encourage a more open communication with the treating oncologist and bring up issues not commonly discussed with the oncologist, such as emotional well-being. Tracking via mobile devices (cell phones and smart phones) provides additional advantages, in that monitoring can occur throughout the day in the person's usual environment.

\section{Lessons flow}

An important decision a developer of an intervention must make is whether to create a sequential, open, or an adaptable lesson flow (lesson plan structure). In a sequential flow, a lesson order is pre-determined; a participant may not "peek ahead" and must complete the lessons in order. The benefit of such an approach is its predictability and the ability of the lessons to build on one another. However, participants may not appreciate their reduced control over the lessons and the need to read several lessons prior to arriving at the desired one, for instance, which may increase dropout. Open lesson plan flow permits participants to view any lesson, in any order. This may be beneficial for participants who desire more control or are only interested in specific topics. The drawbacks include the challenge of creating essentially "free-standing" lessons and reduced predictability of participants' behavior. An adaptable lesson plan flow is structured to suit the needs of a participant, such that the participant sees the most relevant lessons first; the plan itself may be sequential or open. Thus, a cancer survivor struggling with fear of recurrence might see the lesson on this topic prior to all others. In addition, given the high dropout rate of Internet interventions [37,59], the benefit is that the patient is more likely to see the most needed material before dropping out. Adaptable plans require at least some baseline assessment (to gather data to determine lesson order), as well as independent lessons.

\section{Measurable outcomes}

Conducting research on Internet intervention is necessary to establish efficacy and effectiveness; such research is also a powerful scientific tool. Internet intervention studies generate a wealth of data, some of which would not be available in traditional face-to-face trials (e.g., page views, time spent on specific exercises). These data can be used to construct better designed, more efficacious, and more usable future interventions. Unlike traditional treatment outcome studies, which recruit participants from a circumscribed area near the research facility, studies conducted on the Internet are able to recruit large samples of geographically diverse populations $[57,65]$. This allows researchers to answer questions not commonly addressed because of the usual homogeneity of the sample, such as the influence of culture, language, and numerous other demographic and clinical characteristics on clinical outcomes. To accomplish this, Internet interventions need to include validated tools (e.g., standardized questionnaires assessing depression, anxiety, pain, etc., single-item queries, site utilization captures) to determine whether the intervention is usable, utilized, and useful. Some of these data may also be useful to the treating physician to fine-tune the treatment plan or to determine whether a more intensive mental health intervention is needed.

\section{Support emails/messages}

Support emails (or messages, e.g., via SMS) delivered at pre-specified times to intervention users 
might reduce attrition and ultimately produce better outcomes [66-69]. Emails may remind a person to return to and use the site, point to helpful resources, or provide a connection with the treatment team.

\section{Usability testing}

Usability refers to the degree to which an intervention is clear, coherent, and user friendly [70]. Conducting usability testing helps ensure that the website can be navigated and understood by persons of most education levels [70]. Even interventions that include the best current medical knowledge are unlikely to be helpful unless clearly accessible and easy to use. Thorough usability testing is therefore a prerequisite for a successful intervention. Researchers are encouraged to refer to http://www.usability. gov/ for guides on creating Internet content and tools to conduct usability testing. It is highly recommended that all aspects of the intervention be tested vis-à-vis usability, with at least some of the testers being cancer patients with various diagnoses to determine whether an intervention is acceptable for the population toward which it is targeted.

\section{Levels of Internet intervention}

Self-help automated Internet interventions can provide a basic level of health intervention, with the potential for a very wide reach and worldwide dissemination. Communities without other resources would benefit from receiving such evidence-based Internet interventions, rather than nothing. For communities with greater resources, adjunct Internet interventions could also be highly beneficial, assuming, for example, the role of a provider's helper, to reinforce the message of live treatment when such is available. For any forms of computerized intervention, the role of and the need for a live therapist, including the specifics or the "dose" of live therapy, need to be empirically examined, especially in interactions with patient variables (e.g., severity, cancer diagnosis, stage of cancer). It is likely that severe forms of distress may be less amenable to automated interventions, whereas for milder distress, a purely self-help approach may be efficacious and cost-effective. However, these suppositions need to be tested empirically. Some individuals may actually prefer to interact with an infinitely patient self-help automated website that is accessible at all times, from anywhere, and does not impose time limits on the interaction.

\section{Privacy and confidentiality}

Privacy and confidentiality issues online are distinct from those in traditional trials. Participants can avoid potentially embarrassing clinic visit, interactions with staff and providers, and a resulting paper trail. However, they also produce a substantial amount of data, which, if linked to their identifying information and disclosed, may cause harm. Participants utilizing an Internet intervention do and should expect that the information they will share with the site will be kept safely and in confidence. The data gathered by the site are likely to contain sensitive information, as one would share with a service provider. Similar safeguards need to be in place for Internet interventions as exist for any medical data gathered as part of a visit to a physician, or that exist in the personal health record. Limits to confidentiality must be disclosed fully and in a simple-to-understand language, and only the minimum of identifying information should be collected. We recommend that identifiable data be kept in a separate database from the intervention database, linked only by a confidential code. Users should be given access to their data for their own use, or to submit to their healthcare provider.

\section{Provider link}

Designers of Internet interventions delivered as part of the overall health system may choose to include a link between the website and the participants' provider. For instance, an alert might be triggered if the participant's mood becomes markedly worse or if medication non-compliance is noted. However, this ability should be kept in accord with the legal and ethical regulation of transmitting medical information electronically.

\section{Elements shared with face-to-face trials}

Other design considerations for Internet interventions mirror the best practices of face-to-face interventions and should likewise not be overlooked.

\section{Theoretical model of behavior change}

Just as a traditional psychotherapy is guided by its theoretical framework, an Internet intervention must be informed by an appropriate, empirically supported theoretical system of behavior change. Moreover, Internet interventions could contribute to the refinement and testing of theories of health behavior change by subjecting specific elements of the intervention to controlled tests of efficacy. In general and medical populations, CBT interventions have comprised the majority of Internet programs for depression and anxiety. CBT enjoys the strongest empirical support for a variety of psychological problems and disorders. In addition, both the theoretical and practical aspects of CBT are manualized and contain components that rely on a mixture of education and practice, which permits an easier adaptability to nontraditional media. Thus, CBT is a prime example of psychotherapy that could be adapted for online administration for cancer patients and survivors. However, other forms of therapy could conceivably be adapted to online formats (e.g., existential or meaning-centered therapy [71], transtheoretical model communications [72], and Acceptance and Commitment Therapy [73], to name a few). 


\section{Process and structure of the intervention}

A researcher designing a face-to-face trial must decide on the number of sessions, manuals, and the amount of contact with participants. A researcher designing Internet interventions must likewise select the lessons, activities, and tools participants will use and establish the number of reminders, follow-ups, and other aspects of study contact.

\section{Participant selection}

A one-size-fits-all approach is unlikely to succeed given the complexities and the idiosyncrasies of specific cancer diagnoses. For instance, some cancer patients (e.g., breast cancer and head and neck cancer patients) may grapple with body image issues after cancer treatment and may benefit from specific content devoted to these issues. Specific interventions, or a single but highly personalizable intervention, may exist to target specific diagnoses or the diagnosed stage of illness - that is, early stage versus advanced cancer. Internet and mobile app interventions may be particularly appealing to adolescents and young adults in cancer treatment [74]. Internet interventions may also be helpful for cancer survivors who have completed active treatment, helping buffer the perceived loss of support from other patients and providers and facilitating the emotional transition from patient to survivor [75].

\section{Control group}

Researchers designing any trial, Internet or faceto-face, must balance scientific design issues (the need to demonstrate the effect of the intervention) with ethical concerns regarding controls, which are especially relevant in a population as vulnerable and in need of services as cancer patients. Given that attrition in Internet trials can be especially problematic [59], a wait-list control group may not be optimal. Online "control" groups and multiple baseline designs are alternatives to treatment as usual or no

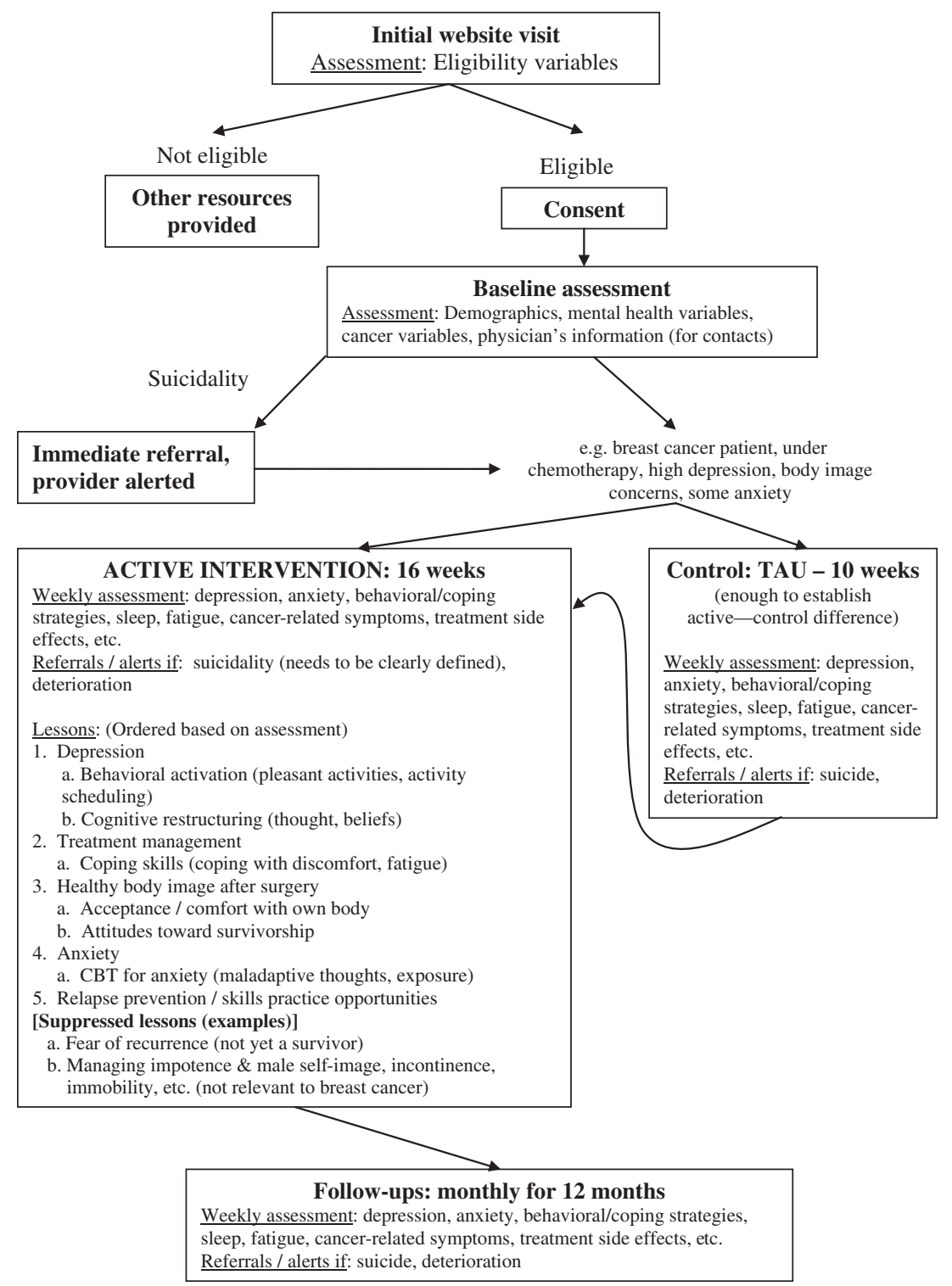

Figure I. An example Internet intervention trial design based on the methodological guidelines 
treatment controls [76]. Given the ease of collecting interval data, time series designs could also be useful for examining how to tailor interventions to individuals.

\section{Summary}

This overview of methodological guidelines should serve as a framework for designing interventions to be delivered wholly, or in part, online. Careful attention to these guidelines will help ensure that results of such intervention studies are as valid and generalizable as possible. In Figure 1, an example trial design based on these guidelines is presented. This illustration is not meant as an ideal or a definitive trial but as an example of choices researchers might make in designing an intervention.

\section{Conclusions}

The prevalence and impact of depression and anxiety in cancer patients demand greater access to mental health interventions. Internet interventions aimed at helping cancer patients and their providers identify and manage the most prevalent emotional symptoms (i.e., depression and anxiety) have the potential to help address the enormous unmet need for mental health services. These interventions, as applied specifically to cancer patients, are worthy of systematic, well-designed, and rigorously conducted studies.

\section{Acknowledgements}

This work was supported, in part, by a funding from the Mt. Zion Health Fund to Dr. Dunn. Dr. Dunn is employed by the University of California, San Francisco and receives funding from the National Institutes of Health. Dr. Leykin is supported by grant K08MH091501, from the National Institute of Mental Health. Dr. Muñoz is supported in part by grant 1R34MH091231 (National Institute of Mental Health).

Conflict of interest: The authors report no conflicts of interest.

\section{References}

1. Institute of Medicine (IOM). Cancer Care for the Whole Patient: Meeting Psychosocial Health Needs. The National Academies Press: Washington, DC, 2007.

2. Zabora J, BrintzenhofeSzoc K, Curbow B, Hooker C, Piantadosi S. The prevalence of psychological distress by cancer site. Psychooncology 2001;10:19-28.

3. Massie MJ. Prevalence of depression in patients with cancer. J Natl Cancer Inst Monogr 2004;32:57-71.

4. Kangas M, Henry JL, Bryant RA. Posttraumatic stress disorder following cancer: a conceptual and empirical review. Clin Psychol Rev 2002;22:499-524.

5. Gotay CC, Pagano IS. Assessment of Survivor Concerns (ASC): a newly proposed brief questionnaire. Health Qual Life Outcomes 2007;5:15.

6. Baker F, Denniston M, Smith T, West MM. Adult cancer survivors: how are they faring? Cancer 2005;104: 2565-2576.

7. Lebel S, Rosberger Z, Edgar L, Devins GM. Comparison of four common stressors across the breast cancer trajectory. J Psychosom Res 2007;63:225-232.

8. Simard S, Savard J. Fear of Cancer Recurrence Inventory: development and initial validation of a multidimensional measure of fear of cancer recurrence. Support Care Cancer 2009; 17:241-251.

9. McDaniel JS, Musselman DL, Porter MR, Reed DA, Nemeroff CB. Depression in patients with cancer: diagnosis, biology, and treatment. Arch Gen Psychiatry 1995;52: 89-99.

10. Kenne Sarenmalm E, Ohlen J, Oden A, Gaston-Johansson F. Experience and predictors of symptoms, distress and health-related quality of life over time in postmenopausal women with recurrent breast cancer. Psychooncology 2008; 17:497-505.

11. Williams S, Dale J. The effectiveness of treatment for depression/depressive symptoms in adults with cancer: a systematic review. Br J Cancer 2006;94:372-390.

12. Manne SL, Andrykowski MA. Are psychological interventions effective and accepted by cancer patients? II. Using empirically supported therapy guidelines to decide. Ann Behav Med 2006;32:98-103.

13. Daniels J, Kissane DW. Psychosocial interventions for cancer patients. Curr Opin Oncol 2008;20:367-371.

14. Andersen BL, Farrar WB, Golden-Kreutz D, et al. Distress reduction from a psychological intervention contributes to improved health for cancer patients. Brain Behav Immun 2007;21:953-961.

15. Osborn RL, Demoncada AC, Feuerstein M. Psychosocial interventions for depression, anxiety, and quality of life in cancer survivors: meta-analyses. Int $\mathrm{J}$ Psychiatry Med 2006;36:13-34

16. Gil KM, Mishel MH, Germino B, Porter LS, Carlton-LaNey I, Belyea M. Uncertainty management intervention for older African American and Caucasian long-term breast cancer survivors. J Psychosoc Oncol 2005;23:3-21.

17. Speca M, Carlson LE, Goodey E, Angen M. A randomized, wait-list controlled clinical trial: the effect of a mindfulness meditation-based stress reduction program on mood and symptoms of stress in cancer outpatients. Psychosom Med 2000;62:613-622.

18. Sheard T, Maguire P. The effect of psychological interventions on anxiety and depression in cancer patients: results of two meta-analyses. Br J Cancer 1999;80:1770-1780.

19. Stanton AL. Psychosocial concerns and interventions for cancer survivors. J Clin Oncol 2006;24:5132-5137.

20. Jacobsen PB, Ransom S. Implementation of NCCN distress management guidelines by member institutions. J Natl Compr Canc Netw 2007;5:99-103.

21. Detmar SB, Muller MJ, Schornagel JH, Wever LD, Aaronson NK. Health-related quality-of-life assessments and patient-physician communication: a randomized controlled trial. J Am Med Assoc 2002;288:3027-3034.

22. McLachlan SA, Allenby A, Matthews J, et al. Randomized trial of coordinated psychosocial interventions based on patient self-assessments versus standard care to improve the psychosocial functioning of patients with cancer. J Clin Oncol 2001;19:4117-4125.

23. Muriel AC, Hwang VS, Kornblith A, et al. Management of psychosocial distress by oncologists. Psychiatr Serv 2009;60:1132-1134.

24. Munoz RF. Using evidence-based Internet interventions to reduce health disparities worldwide. J Med Internet Res 2010;12:e60.

25. Helft PR. A new age for cancer information seeking: are we better off now? J Gen Intern Med 2008;23:350-352.

26. Stark D, Kiely M, Smith A, et al. Reassurance and the anxious cancer patient. Br J Cancer 2004;91:893-899.

27. Chou WY, Hunt YM, Beckjord EB, Moser RP, Hesse BW. Social media use in the United States: implications for health communication. J Med Internet Res 2009; 11:e48.

28. Lieberman MA, Goldstein BA. Self-help on-line: an outcome evaluation of breast cancer bulletin boards. J Health Psychol 2005;10:855-862. 
29. Hoybye MT, Dalton SO, Deltour I, Bidstrup PE, Frederiksen K, Johansen C. Effect of Internet peer-support groups on psychosocial adjustment to cancer: a randomised study. Br J Cancer 2010;102:1348-1354.

30. Klemm P, Bunnell D, Cullen M, Soneji R, Gibbons P, Holecek A. Online cancer support groups: a review of the research literature. Comput Inform Nurs 2003;21:136-142.

31. Clarke G, Eubanks D, Reid E, et al. Overcoming Depression on the Internet (ODIN) (2): a randomized trial of a self-help depression skills program with reminders. $J$ Med Internet Res 2005;7:e16.

32. Marks I, Cavanagh K. Computer-aided psychological treatments: evolving issues. Annu Rev Clin Psychol 2009; 5:121-141.

33. Berger $\mathrm{M}$, Wagner $\mathrm{TH}$, Baker LC. Internet use and stigmatized illness. Soc Sci Med 2005;61:1821-1827.

34. Owen JE, Klapow JC, Roth DL, Nabell L, Tucker DC. Improving the effectiveness of adjuvant psychological treatment for women with breast cancer: the feasibility of providing online support. Psychooncology 2004; 13:281-292.

35. Strecher V. Internet methods for delivering behavioral and health-related interventions (eHealth). Аnnu Rev Clin Psychol 2007;3:53-76.

36. McCarrier KP, Ralston JD, Hirsch IB, et al. Web-based collaborative care for type 1 diabetes: a pilot randomized trial. Diab Technol Ther 2009;11:211-217.

37. Christensen H, Griffiths K, Groves C, Korten A. Free range users and one hit wonders: community users of an Internetbased cognitive behaviour therapy program. Aust New Zeal J Psychiatr 2006;40:59-62.

38. Glasgow RE, Vogt TM, Boles SM. Evaluating the public health impact of health promotion interventions: the REAIM framework. Am J Public Health 1999;89:1322-1327.

39. Andrykowski MA, Burris JL. Use of formal and informal mental health resources by cancer survivors: differences between rural and nonrural survivors and a preliminary test of the theory of planned behavior. Psychooncology 2010;19:1148-1155.

40. Munoz RF, Barrera AZ, Delucchi K, Penilla C, Torres LD, Perez-Stable EJ. International Spanish/English Internet smoking cessation trial yields $20 \%$ abstinence rates at 1 year. Nicotine Tob Res 2009;11:1025-1034.

41. Internet World Stats. United States of America: Internet usage and broadband usage report. 2010.

42. van Bastelaar KM, Pouwer F, Cuijpers P, Twisk JW, Snoek FJ. Web-based cognitive behavioural therapy (W-CBT) for diabetes patients with co-morbid depression: design of a randomised controlled trial. BMC Psychiatry 2008;8:9.

43. Warmerdam L, van Straten A, Twisk J, Riper H, Cuijpers P. Internet-based treatment for adults with depressive symptoms: randomized controlled trial. J Med Internet Res 2008; 10:e44.

44. Lustria ML, Cortese J, Noar SM, Glueckauf RL. Computertailored health interventions delivered over the Web: review and analysis of key components. Patient Educ Counsel 2009; 74:156-173.

45. Griffiths KM, Farrer L, Christensen H. The efficacy of Internet interventions for depression and anxiety disorders: a review of randomised controlled trials. Med J Aust 2010;192:S4-S11.

46. Winzelberg AJ, Classen C, Alpers GW, et al. Evaluation of an Internet support group for women with primary breast cancer. Cancer 2003;97:1164-1173.

47. Owen JE, Klapow JC, Roth DL, et al. Randomized pilot of a self-guided Internet coping group for women with earlystage breast cancer. Ann Behav Med 2005;30:54-64.

48. Dumrongpakapakorn P, Hopkins K, Sherwood P, Zorn K, Donovan H. Computer-mediated patient education: opportunities and challenges for supporting women with ovarian cancer. Nurs Clin North Am 2009;44:339-354.
49. Kroenke K, Theobald D, Wu J, et al. Effect of telecare management on pain and depression in patients with cancer: a randomized trial. J Am Med Assoc 2010;304: 163-171.

50. Ritterband L. Improving the sleep of cancer patients using an Internet-based program (SHUTi-C). 2011 (Available from: http://www.shuti.net [Accessed 14 Dec 2010]).

51. Griffiths KM, Calear AL, Banfield M. Systematic review on Internet Support Groups (ISGs) and depression (1): do ISGs reduce depressive symptoms? J Med Internet Res 2009;11:e40.

52. Salzer MS, Palmer SC, Kaplan K, et al. A randomized, controlled study of Internet peer-to-peer interactions among women newly diagnosed with breast cancer. PsychoOncology 2010;19:441-446.

53. Tolin DF. Is cognitive-behavioral therapy more effective than other therapies? A meta-analytic review. Clin Psychol Rev 2010;30:710-720.

54. Stewart RE, Chambless DL. Cognitive-behavioral therapy for adult anxiety disorders in clinical practice: a metaanalysis of effectiveness studies. J Consult Clin Psychol 2009;77:595-606.

55. Butler AC, Chapman JE, Forman EM, Beck AT. The empirical status of cognitive-behavioral therapy: a review of meta-analyses. Clin Psychol Rev 2006;26: $17-31$.

56. Crits-Christoph P. The efficacy of brief dynamic psychotherapy: a meta-analysis. Am J Psychiatry 1992;149: 151-158.

57. Ritterband LM, Andersson G, Christensen HM, Carlbring P, Cuijpers P. Directions for the International Society for Research on Internet Interventions (ISRII). J Med Internet Res 2006;8:e23.

58. Cuijpers $P$, van Straten A, Andersson G. Internet-administered cognitive behavior therapy for health problems: a systematic review. J Behav Med 2008;31:169-177.

59. Eysenbach G. The law of attrition. J Med Internet Res 2005;7:e11

60. Barak A, Klein B, Proudfoot JG. Defining Internetsupported therapeutic interventions. Ann Behav Med 2009;38:4-17.

61. Burns DD, Nolen-Hoeksema S. Coping styles, homework compliance, and the effectiveness of cognitive-behavioral therapy. J Consult Clin Psychol 1991;59:305-311.

62. Garland A, Scott J. Using homework in therapy for depression. J Clin Psychol 2002;58:489-498.

63. Strecher VJ, Shiffman S, West R. Moderators and mediators of a Web-based computer-tailored smoking cessation program among nicotine patch users. Nicotine Tob Res 2006;8(Suppl 1):S95-S101.

64. Hermansen-Kobulnicky CJ. Symptom-monitoring behaviors of rural cancer patients and survivors. Support Care Cancer 2009;17:617-626.

65. Barrera AZ, Perez-Stable EJ, Delucchi KL, Munoz RF. Global reach of an Internet smoking cessation intervention among Spanish- and English-speaking smokers from 157 countries. Int J Environ Res Public Health 2009;6: 927-940.

66. Lenert L, Muñoz RF, Stoddard J, et al. Design and pilot evaluation of an Internet smoking cessation program. $J \mathrm{Am}$ Med Inform Assoc 2003; 10:16-20.

67. Lenert L, Muñoz RF, Perez JE, Bansod A. Automated e-mail messaging as a tool for improving quit rates in an Internet smoking cessation intervention. $\mathrm{J} \mathrm{Am} \mathrm{Med}$ Inform Assoc 2004:11:235-240.

68. Muñoz RF, Lenert LL, Delucchi K, et al. Toward evidencebased Internet interventions: a Spanish/English Web site for international smoking cessation trials. Nicotine Tob Res 2006;8:77-87.

69. Van Osch L, Lechner L, Reubsaet A, Steenstra M, Wigger S, De Vries H. Optimizing the efficacy of smoking cessation 
contests: an exploration of determinants of successful quitting. Health Educ Res 2009;24:54-63.

70. Stoddard JL, Augustson EM, Mabry PL. The importance of usability testing in the development of an Internet-based smoking cessation treatment resource. Nicotine Tob Res 2006;8:87-93.

71. Kissane DW, Bloch S, Miach P, Smith GC, Seddon A, Keks N. Cognitive-existential group therapy for patients with primary breast cancer: techniques and themes. Psychooncology 1997;6:25-33.

72. Prochaska JO, Butterworth S, Redding CA, et al. Initial efficacy of MI, TTM tailoring and HRI's with multiple behaviors for employee health promotion. Prev Med 2008;46:226-231.
73. Ost LG. Efficacy of the third wave of behavioral therapies: a systematic review and meta-analysis. Behav Res Ther 2008;46:296-321.

74. Tercyak KP, Abraham AA, Graham AL, Wilson LD, Walker LR. Association of multiple behavioral risk factors with adolescents' willingness to engage in eHealth promotion. J Pediatr Psychol 2009;34:457-469.

75. Meneses KD, McNees P, Loerzel VW, Su X, Zhang Y, Hassey LA. Transition from treatment to survivorship: effects of a psychoeducational intervention on quality of life in breast cancer survivors. Oncol Nurs Forum 2007;34:1007-1016.

76. Danaher BG, Seeley JR. Methodological issues in research on Web-based behavioral interventions. Ann Behav Med 2009;38:28-39. 